\title{
Genetic Variability and Association Analysis of Maize Hybrids under Excessive Soil Moisture Condition
}

\author{
Modekurti Sravana Lakshmi ${ }^{1}$, Prem Narayan Jagadev ${ }^{1}$, Sindhusnata Das ${ }^{1}$, \\ Devraj Lenka ${ }^{1}$, Digvijay Swain ${ }^{1}$ and Swapan K. Tripathy ${ }^{2^{*}}$
}
${ }^{1}$ Department of Plant Breeding and Genetics, ${ }^{2}$ Department of Agricultural Biotechnology, College of Agriculture, OUAT, Bhubaneswar, India

*Corresponding author

\begin{abstract}
A B S T R A C T
Excess Soil Moisture (ESM) stress caused by temporary water logging is a major limiting factor for maize production and productivity in Asian countries. In this context, the present

Keywords

Genetic variability,

Character association,

Path analysis, Water

logging stress, Zea mays L.

Article Info

Accepted:

20 August 2018

Available Online:

10s September 2018 investigation was carried out to study genetic variability, character association and path analysis in 35 maize hybrids under excessive soil moisture condition. Analysis of variance revealed highly significant differences among the genotypes for all the characters. High heritability with high genetic advance was observed in senescence percent(after excess water stress), difference in senescence, number of kernels per row, number of kernels per cob and grain yield, indicating the preponderance of additive gene action in controlling the traits. Yield was positively and significantly correlated with plant height, ear height, length of cob, girth of cob, number of kernel rows per cob, number of kernels per row, number of kernels per cob, 100 kernel weight and shelling \%. Among these, number of kernels per row, days to $50 \%$ tasseling, 100 kernel weight and number of kernels per cob had direct positive effect on grain yield at genotypic level. Senescence percent had shown highest positive indirect effect via number of kernels per row on grain yield. This happens to be the case in water logging tolerant hybrids.
\end{abstract}

\section{Introduction}

Maize (Zea mays L.), also known as corn, is the third most important cereal crop in India next to rice and wheat. Among various abiotic stresses, Excess Soil Moisture (ESM) stress caused by temporary water logging due to heavy rains. Low pressure followed by monsoon makes the fields saturated. Water logging is one of the most important constraints for maize production and productivity in Asian regions. In India, about 8.5 million ha of arable soil is affected.

Out of the 6.6 million ha total area of maize, about 2.5 million ha is exposed to excess soil moisture that causes on average 25-30\% loss of national maize production almost every year (DMR, 2001). Keeping in view the present condition and facts, the present 
investigation was undertaken to identify hybrids tolerant to water logging stress.

\section{Materials and Methods}

The present study was about evaluation of 35 single cross maize hybrids (Zea mays L) laid out in a randomized complete block design (RCBD) with two replications at EB-II section of Department of Plant Breeding and Genetics, College of Agriculture, OUAT, Bhubaneswar during KHARIF 2016-17. Waterlogging stress was imposed for 7 days from the beginning of flowering stage by maintaining about10-12 centimetres of water level in the field (water stagnation). Proper bunding was done so that water remains within. And after 7 days, water was drained out. Subsequently, different morphological observations viz number of leaves, leaf senescence percent (after stress), difference in senescence percent (after stress - before stress) along with yield and other morphoeconomic traits were recorded. Analysis of variance, correlation and path coefficient studies were undertaken.

\section{Results and Discussion}

\section{Study of genetic variability}

In the table 1, the analysis of variance for sixteen characters under excessive soil moisture condition had been presented. From f-test, it was revealed there were significant differences among the hybrids for all the characters under investigation.

\section{Phenotypic and genotypic coefficient of variation}

The estimates of parameters of genetic variability such as mean, range, PCV, GCV, heritability $\left(\mathrm{h}^{2}{ }_{\mathrm{bs}}\right)$ and expected genetic advance from selection were presented in table 2. The estimates of phenotypic variability was low (below $10 \%$ ) for characters like days to 50 per cent tasseling, days to 50 per cent silking, days to 75 per cent dry husk, plant height, number of leaves, number of kernel rows per cob and shelling per cent. The estimates of PCV were moderate (10-20\%) for ear height, length of cob, girth of cob, 100 kernel weight. These estimates were high (> $20 \%$ ) for senescence per cent (after stress), difference in senescence per cent, number of kernels per row, number of kernels per cob and yield. GCV estimates also showed similar results.

\section{Heritability and genetic advance}

Characters like number of leaves $(60.63 \%)$ exhibited moderate heritability (40\%-70\%) and all other characters exhibited high heritability $(>70 \%)$ indicating that selection of these characters were useful for further generation in improvement of breeding programme. Such similar results were reported by F. Nzuve et al., 2014 for the characters like yield, ear and plant height and also by Yogesh Pandey et al., 2017 for the characters like yield, shelling \% and 100 kernel weight.

Expected genetic advance was low (0-15\%) in case of days to 50 per cent tasseling, days to 50 per cent silking, days to 75 per cent dry husk, plant height, number of leaves, length of cob, number of kernel rows per cob and shelling per cent. Moderate (15-30\%) genetic advance was reported for ear height, girth of cob and100 kernel weight. Characters like senescence per cent (after stress), difference in senescence per cent, number of kernels per row, number of kernels per cob and yield showed high (> 30\%) genetic advance.

Characters like days to 50 per cent tasseling, days to 50 per cent tasseling, days to 50 per cent silking, days to 75 per cent dry husk, plant height, length of cob, number of kernel rows per cob and shelling per cent observed 
high heritability accompanied with low genetic advance indicating that the expression of such traits was under the control of nonadditive type of gene action, and its response to selection would be poor. High heritability with moderate genetic advance was observed in ear height, girth of cob and 100 kernel weight suggesting that the expression of these traits was mostly influenced by additive type of gene action and its response to selection would be effective in improving the seed yield. High heritability accompanied with high genetic advance was observed in senescence percent (after stress), difference in senescence percent, number of kernels per row, number of kernels per cob and yield which indicated the preponderance of additive gene action in controlling the traits.

Table.1 ANOVA (RBD) for 16 agro-economic traits in 35 maize hybrids

\begin{tabular}{|c|c|c|c|c|}
\hline \multirow[t]{2}{*}{ Sl. No } & \multirow[t]{2}{*}{ Character } & \multicolumn{3}{|c|}{ Mean Sum of Squares } \\
\hline & & Replication (1) & Genotype (34) & Error (34) \\
\hline 1 & Days to $50 \%$ tasseling (DT) & 0.36 & $14.74^{* *}$ & 0.33 \\
\hline 2 & Days to $50 \%$ silking (DS) & 0.91 & $14.87^{* *}$ & 0.70 \\
\hline 3 & Days to $75 \%$ dry husk (DH) & 0.24 & $4.19^{* *}$ & 0.38 \\
\hline 4 & Plant height $(\mathrm{cm})(\mathrm{PHT})$ & 52.83 & $211.89^{* *}$ & 27.17 \\
\hline 5 & Ear height $(\mathrm{cm})(\mathrm{EH})$ & 0.06 & $190.59^{* *}$ & 28.30 \\
\hline 6 & Number of leaves (NL) & $2.13^{*}$ & $0.94^{* *}$ & 0.37 \\
\hline 7 & Senescence per cent (After Stress) (\%) (SP) & 9.09 & $139.31^{* *}$ & 23.72 \\
\hline 8 & Difference in senescence per cent (\%) (DSP) & 19.67 & $81.03^{* *}$ & 6.55 \\
\hline 9 & Length of cob (cm) (LC) & 0.05 & $5.87^{* *}$ & 1.58 \\
\hline 10 & Girth of $\operatorname{cob}(\mathrm{cm})(\mathrm{GC})$ & 1.03 & $3.98^{* *}$ & 0.44 \\
\hline 11 & Number of kernel rows per cob $(\mathrm{KR} / \mathrm{C})$ & $1.64^{*}$ & $2.11^{* * *}$ & 0.36 \\
\hline 12 & Number of kernels per row (K/R) & 0.002 & $62.66^{* *}$ & 5.62 \\
\hline 13 & Number of kernels per cob (KN/C) & 948.46 & $16771.15^{* *}$ & 1471.05 \\
\hline 14 & 100 kernel weight $(\mathrm{g})(\mathrm{KW})$ & $35.17^{* *}$ & $19.48^{* *}$ & 3.79 \\
\hline 15 & Shelling \% (S) & 0.40 & $26.99^{* *}$ & 6.73 \\
\hline 16 & Yeild (q/ha) (Y) & $2273.09^{* *}$ & $864.21^{* *}$ & 63.79 \\
\hline
\end{tabular}

Table.2 Genetic parameters of variability for grain yield in 35 maize hybrids

\begin{tabular}{|r|l|c|c|c|c|c|c|c|}
\hline $\begin{array}{l}\text { Sl. } \\
\text { No. }\end{array}$ & Characters & Mean & Range & $\begin{array}{c}\text { PCV } \\
(\%)\end{array}$ & $\begin{array}{c}\text { GCV } \\
(\%)\end{array}$ & $\begin{array}{c}\mathbf{h}^{2} \mathbf{b} \\
(\mathbf{\%})\end{array}$ & Gs 10 & Gs \% \\
\hline $\mathbf{1}$ & Days to 50\% tasseling & 55.30 & $49.00-63.00$ & 4.91 & 4.85 & 97.78 & 4.67 & 8.45 \\
\hline $\mathbf{2}$ & Days to 50\% silking & 55.49 & $49.00-63.00$ & 4.91 & 4.80 & 95.43 & 4.58 & 8.25 \\
\hline $\mathbf{3}$ & Days to 75\% dry husk & 85.91 & $81.00-81.50$ & 1.68 & 1.61 & 91.05 & 2.32 & 2.70 \\
\hline $\mathbf{4}$ & Plant height (cm) & 169.40 & $147.50-190.50$ & 6.08 & 5.67 & 87.18 & 15.79 & 9.32 \\
\hline $\mathbf{5}$ & Ear height (cm) & 68.81 & $47.30-86.20$ & 14.19 & 13.09 & 85.15 & 14.63 & 21.26 \\
\hline $\mathbf{6}$ & Number of leaves & 13.83 & $11.80-15.10$ & 4.96 & 3.86 & 60.63 & 0.73 & 5.29 \\
\hline $\mathbf{7}$ & Senescence percent (After stress) (\%) & 32.67 & $21.39-54.53$ & 25.55 & 23.27 & 82.98 & 12.19 & 37.31 \\
\hline $\mathbf{8}$ & Difference in senescence percent & 18.74 & $11.43-41.40$ & 33.96 & 32.56 & 91.92 & 10.30 & 54.94 \\
\hline $\mathbf{9}$ & Length of cob (cm) & 15.27 & $11.98-17.60$ & 11.21 & 9.58 & 73.07 & 2.20 & 14.42 \\
\hline $\mathbf{1 0}$ & Girth of cob (cm) & 13.14 & $10.37-15.54$ & 10.74 & 10.13 & 89.06 & 2.21 & 16.83 \\
\hline $\mathbf{1 1}$ & No. of kernel rows per cob & 13.83 & $10.80-16.00$ & 7.43 & 6.77 & 83.05 & 1.50 & 10.86 \\
\hline $\mathbf{1 2}$ & No. of kernels per row & 25.04 & $15.35-35.00$ & 22.35 & 21.32 & 91.02 & 8.97 & 35.80 \\
\hline $\mathbf{1 3}$ & No. of kernels per cob & 357.22 & $197.35-548.50$ & 25.64 & 24.49 & 91.23 & 147.03 & 41.16 \\
\hline $\mathbf{1 4}$ & 100 kernel weight (g) & 26.39 & $18.39-33.22$ & 11.82 & 10.61 & 80.56 & 4.42 & 16.76 \\
\hline $\mathbf{1 5}$ & Shelling \% & 80.94 & $73.65-87.35$ & 4.54 & 3.93 & 75.08 & 4.85 & 6.00 \\
\hline $\mathbf{1 6}$ & Yeild (q/ha) & 41.48 & $13.80-80.65$ & 50.11 & 48.23 & 92.62 & 33.88 & 81.68 \\
\hline
\end{tabular}


Table.3 Phenotypic $\left(r_{\mathrm{p}}\right)$ and genotypic $\left(\mathrm{r}_{\mathrm{g}}\right)$ correlation coefficient among 16 characters in 35 maize hybrids

\begin{tabular}{|c|c|c|c|c|c|c|c|c|c|c|c|c|c|c|c|c|}
\hline Characters & Correl & DS & DH & PHT & EH & $\mathbf{L N}$ & SP & DSP & LC & GC & $\mathrm{KR} / \mathrm{C}$ & $\mathbf{K} / \mathbf{R}$ & $\mathrm{KN} / \mathrm{C}$ & KW & $\mathbf{S} \%$ & $\mathbf{Y}$ \\
\hline \multirow[t]{2}{*}{ DT } & $r_{p}$ & $0.982^{* *}$ & 0.141 & $-0.370^{*}$ & $0.382^{*}$ & -0.070 & $0.472^{* *}$ & $0.357^{*}$ & $-0.470^{* *}$ & $-0.480^{* *}$ & -0.125 & $-0.552^{* *}$ & $-0.510^{* *}$ & $-0.543^{* *}$ & $-0.621^{* *}$ & $-0.593^{* *}$ \\
\hline & $r_{g}$ & $0.992^{* *}$ & 0.133 & $-0.393^{*}$ & $-0.407^{*}$ & -0.073 & $0.540^{* *}$ & $0.392^{*}$ & $-0.559^{* *}$ & $-0.530^{* *}$ & -0.142 & $-0.575^{* *}$ & $-0.540^{* *}$ & $-0.630^{* *}$ & $-0.730^{* *}$ & $-0.616^{* *}$ \\
\hline \multirow[t]{2}{*}{$\overline{\text { DS }}$} & $r_{p}$ & & 0.147 & $-0.370^{*}$ & $-0.394^{*}$ & -0.074 & $0.486^{* *}$ & $0.381^{*}$ & $-0.465^{* *}$ & $-0.492^{* *}$ & -0.120 & $-0.556^{* *}$ & $-0.506^{* *}$ & $-0.540^{* *}$ & $-0.657^{* *}$ & $-0.577^{* *}$ \\
\hline & $\mathrm{r}_{\mathrm{g}}$ & & 0.134 & $-0.397^{*}$ & $-0.419^{*}$ & -0.069 & $0.564^{* *}$ & $0.422^{*}$ & $-0.579^{* *}$ & $-0.540^{* *}$ & -0.123 & $-0.580^{* *}$ & $-0.536^{* *}$ & $-0.624^{* *}$ & $-0.786^{* *}$ & $-0.602^{* *}$ \\
\hline \multirow[t]{2}{*}{ DH } & $r_{p}$ & & & 0.212 & 0.280 & 0.102 & -0.047 & -0.008 & 0.302 & 0.275 & $0.353^{*}$ & 0.167 & 0.203 & 0.091 & 0.228 & 0.073 \\
\hline & $r_{g}$ & & & 0.241 & 0.302 & 0.125 & -0.037 & -0.010 & $0.386^{*}$ & 0.296 & $0.445^{* *}$ & 0.181 & 0.217 & 0.120 & 0.268 & 0.099 \\
\hline \multirow[t]{2}{*}{ PHT } & $r_{p}$ & & & & $0.880^{* *}$ & $0.357^{*}$ & $-0.651^{* *}$ & $-0.631^{* *}$ & $0.671^{* *}$ & $0.640^{* *}$ & $0.500^{* *}$ & $0.727^{* *}$ & $0.736^{* *}$ & $0.447^{* *}$ & $0.618^{* *}$ & $0.656^{* *}$ \\
\hline & $\mathrm{r}_{\mathrm{g}}$ & & & & $0.900^{* *}$ & $0.353^{*}$ & $-0.741^{* *}$ & $-0.738^{* *}$ & $0.774^{* *}$ & $0.694^{* *}$ & $0.562^{* *}$ & $0.817^{* *}$ & $0.834^{* *}$ & $0.552^{* *}$ & $0.806^{* *}$ & 0.736 ** \\
\hline \multirow[t]{2}{*}{ EH } & $r_{p}$ & & & & & 0.238 & $-0.698^{* *}$ & $-0.626^{* *}$ & $0.652^{* *}$ & $0.632^{* *}$ & $0.427^{*}$ & $0.687^{* *}$ & $0.688^{* *}$ & $0.414^{*}$ & $0.635^{* *}$ & $0.632^{* * *}$ \\
\hline & $r_{g}$ & & & & & 0.170 & $-0.815^{* *}$ & $-0.754^{* *}$ & $0.751^{* *}$ & $0.690^{* *}$ & $0.481^{* *}$ & $0.756^{* *}$ & $0.769^{* *}$ & $0.463^{* *}$ & $0.831^{* *}$ & $0.690^{* *}$ \\
\hline \multirow[t]{2}{*}{$\overline{\mathbf{L N}}$} & $r_{p}$ & & & & & & -0.227 & -0.175 & 0.278 & $0.336^{*}$ & $0.489^{* *}$ & $0.367^{*}$ & $0.409^{*}$ & 0.032 & $0.359^{*}$ & 0.288 \\
\hline & $r_{g}$ & & & & & & -0.296 & -0.294 & 0.318 & $0.394^{*}$ & $0.613^{* *}$ & $0.457^{* *}$ & $0.531^{* *}$ & -0.022 & $0.577^{* *}$ & $0.352^{*}$ \\
\hline SP & $r_{p}$ & & & & & & & $0.938^{* *}$ & $-0.658^{* *}$ & $-0.729^{* *}$ & $-0.345^{*}$ & $-0.623^{* *}$ & $-0.628^{* *}$ & $-0.512^{* *}$ & $-0.456^{* *}$ & $-0.703^{* *}$ \\
\hline \multirow[t]{2}{*}{ DSP } & $r_{p}$ & & & & & & & & $-0.595^{* *}$ & $-0.634^{* * *}$ & -0.329 & $-0.541^{* *}$ & $-0.552^{* * *}$ & $-0.403^{* *}$ & $-0.363^{*}$ & $-0.640^{* * *}$ \\
\hline & $\mathrm{r}_{\mathrm{g}}$ & & & & & & & & $-0.733^{* *}$ & $-0.679^{* *}$ & $-0.374^{*}$ & $-0.613^{* *}$ & $-0.611^{* *}$ & $-0.463^{* *}$ & $-0.438^{* *}$ & $-0.681^{* *}$ \\
\hline \multirow[t]{2}{*}{$\mathrm{LC}$} & $r_{p}$ & & & & & & & & & $0.794^{* *}$ & $0.558^{* * *}$ & $0.837^{* *}$ & $0.883^{* *}$ & $0.506^{* *}$ & $0.584^{* *}$ & $0.769^{* *}$ \\
\hline & $r_{g}$ & & & & & & & & & $0.885^{* *}$ & $0.638^{* *}$ & $0.944^{* *}$ & $1.038^{* *}$ & $0.591^{* *}$ & $0.729^{* *}$ & $0.884^{* *}$ \\
\hline \multirow[t]{2}{*}{$\mathbf{G C}$} & $r_{p}$ & & & & & & & & & & $0.658^{* *}$ & $0.827^{* *}$ & $0.858^{* *}$ & $0.719^{* *}$ & $0.700^{* *}$ & $0.835^{* *}$ \\
\hline & $r_{g}$ & & & & & & & & & & $0.702^{* *}$ & $0.869^{* *}$ & $0.907^{* *}$ & $0.777^{* *}$ & $0.785^{* *}$ & $0.910^{* *}$ \\
\hline \multirow[t]{2}{*}{$\mathrm{KR} / \mathrm{C}$} & $r_{p}$ & & & & & & & & & & & $0.519^{* *}$ & $0.664^{* *}$ & $0.344^{*}$ & $0.433^{* *}$ & $0.441^{* *}$ \\
\hline & $r_{g}$ & & & & & & & & & & & $0.579^{* *}$ & $0.723^{* *}$ & $0.385^{*}$ & $0.479^{* *}$ & $0.447^{* *}$ \\
\hline \multirow[t]{2}{*}{$\mathbf{K} / \mathbf{R}$} & $r_{p}$ & & & & & & & & & & & & $0.971^{* *}$ & $0.490^{* *}$ & $0.789^{* *}$ & $0.899^{* *}$ \\
\hline & $r_{g}$ & & & & & & & & & & & & $0.984^{* *}$ & $0.524^{* *}$ & $0.846^{* *}$ & $0.965^{* *}$ \\
\hline \multirow[t]{2}{*}{$\mathrm{KN} / \mathrm{C}$} & $r_{p}$ & & & & & & & & & & & & & $0.486^{* *}$ & $0.750^{* *}$ & $0.882^{* *}$ \\
\hline & $r_{g}$ & & & & & & & & & & & & & $0.530^{* *}$ & $0.796^{* *}$ & $0.944^{* * *}$ \\
\hline \multirow[t]{2}{*}{ KW } & $r_{p}$ & & & & & & & & & & & & & & $0.552^{* *}$ & $0.557^{* *}$ \\
\hline & $r_{g}$ & & & & & & & & & & & & & & $0.665^{* *}$ & $0.605^{* *}$ \\
\hline \multirow[t]{2}{*}{ S\% } & $r_{p}$ & & & & & & & & & & & & & & & $0.752^{* * *}$ \\
\hline & $r_{g}$ & & & & & & & & & & & & & & & $0.877^{* *}$ \\
\hline
\end{tabular}

$r \geq 0.334$ sig at $5 \%$ level, $r \geq 0.429$ sig at $1 \%$ level 
Table.4 Phenotypic $\left(\mathrm{p}_{\mathrm{p}}\right)$ and genotypic $\left(\mathrm{p}_{\mathrm{g}}\right)$ path coefficient analysis showing direct and indirect effects of different traits on yield of 35 hybrids

\begin{tabular}{|c|c|c|c|c|c|c|c|c|c|c|c|c|c|c|c|c|c|}
\hline Character & $\begin{array}{l}\text { Path } \\
\text { coeff. }\end{array}$ & DT & DS & DH & PHT & EH & $\mathbf{L N}$ & SP & DSP & $\mathrm{LC}$ & GC & $\mathrm{KR} / \mathrm{C}$ & $\mathbf{K} / \mathbf{R}$ & $\mathrm{KN} / \mathrm{C}$ & KW & $\mathbf{S} \%$ & Corr. with yield \\
\hline \multirow[t]{2}{*}{ DT } & $\mathrm{p}_{\mathrm{p}}$ & -1.064 & 1.104 & -0.014 & 0.031 & 0.017 & 0.003 & 0.058 & -0.122 & 0.015 & -0.178 & 0.027 & -0.068 & -0.218 & 0.030 & -0.213 & $-0.593^{* *}$ \\
\hline & $\mathrm{p}_{\mathrm{g}}$ & 1.155 & -1.036 & 0.015 & 0.113 & -0.052 & -0.019 & -0.800 & 0.450 & 0.031 & 0.411 & 0.019 & -0.714 & -0.205 & -0.338 & 0.354 & $-0.616^{* *}$ \\
\hline \multirow[t]{2}{*}{$\overline{\mathrm{DS}}$} & $\mathrm{p}_{\mathrm{p}}$ & -1.045 & 1.125 & -0.015 & 0.031 & 0.017 & 0.003 & 0.060 & -0.130 & 0.015 & -0.182 & 0.026 & -0.068 & -0.217 & 0.030 & -0.225 & $-0.577^{* *}$ \\
\hline & $\mathrm{p}_{\mathrm{g}}$ & 1.146 & -1.044 & 0.015 & 0.114 & -0.054 & -0.018 & -0.835 & 0.484 & 0.032 & 0.419 & 0.017 & -0.720 & -0.203 & -0.335 & 0.381 & $-0.602^{* *}$ \\
\hline \multirow[t]{2}{*}{$\overline{\text { DH }}$} & $\mathrm{p}_{\mathrm{p}}$ & -0.150 & 0.165 & -0.102 & -0.018 & -0.012 & -0.004 & -0.006 & 0.003 & -0.009 & 0.102 & -0.076 & 0.021 & 0.087 & -0.005 & 0.078 & 0.073 \\
\hline & $\mathrm{p}_{\mathrm{g}}$ & 0.154 & -0.140 & 0.110 & -0.069 & 0.039 & 0.032 & 0.055 & -0.011 & -0.021 & -0.230 & -0.061 & 0.225 & 0.082 & 0.064 & -0.130 & 0.099 \\
\hline \multirow[t]{2}{*}{ PHT } & $\mathrm{p}_{\mathrm{p}}$ & 0.394 & -0.416 & -0.022 & -0.083 & -0.038 & -0.013 & -0.080 & 0.215 & -0.021 & 0.237 & -0.108 & 0.090 & 0.315 & -0.025 & 0.212 & $0.656^{* *}$ \\
\hline & $\mathrm{p}_{\mathrm{g}}$ & -0.454 & 0.414 & 0.027 & -0.287 & 0.115 & 0.091 & 1.098 & -0.847 & -0.043 & -0.539 & -0.077 & 1.015 & 0.316 & 0.297 & -0.391 & $0.736^{* *}$ \\
\hline \multirow[t]{2}{*}{$\overline{\text { EH }}$} & $\mathrm{p}_{\mathrm{p}}$ & 0.407 & -0.443 & -0.029 & -0.073 & -0.044 & -0.009 & -0.086 & 0.214 & -0.020 & 0.234 & -0.092 & 0.085 & 0.294 & -0.023 & 0.218 & $0.632^{* *}$ \\
\hline & $\mathrm{p}_{\mathrm{g}}$ & -0.470 & 0.437 & 0.033 & -0.258 & 0.128 & 0.044 & 1.207 & -0.865 & -0.041 & -0.536 & -0.066 & 0.939 & 0.292 & 0.249 & -0.403 & $0.690^{* *}$ \\
\hline \multirow[t]{2}{*}{$\overline{L N}$} & $\mathrm{p}_{\mathrm{p}}$ & 0.075 & -0.083 & -0.010 & -0.030 & -0.010 & -0.036 & -0.028 & 0.060 & -0.009 & 0.124 & -0.106 & 0.045 & 0.175 & -0.002 & 0.123 & 0.288 \\
\hline & $\mathrm{p}_{\mathrm{g}}$ & -0.084 & 0.072 & 0.014 & -0.101 & 0.022 & 0.259 & 0.438 & -0.337 & -0.017 & -0.306 & -0.084 & 0.567 & 0.201 & -0.012 & -0.280 & $0.352^{*}$ \\
\hline \multirow[t]{2}{*}{ SP } & $\mathrm{p}_{\mathrm{p}}$ & -0.502 & 0.547 & 0.005 & 0.054 & 0.030 & 0.008 & 0.124 & -0.320 & 0.021 & -0.270 & 0.075 & -0.077 & -0.269 & 0.029 & -0.156 & $-0.703^{* *}$ \\
\hline & $\mathrm{p}_{\mathrm{g}}$ & 0.624 & -0.589 & -0.004 & 0.213 & -0.104 & -0.077 & -1.481 & 1.137 & 0.040 & 0.616 & 0.051 & -0.877 & -0.269 & -0.336 & 0.274 & $-0.782^{* *}$ \\
\hline \multirow[t]{2}{*}{ DSP } & $\mathrm{p}_{\mathrm{p}}$ & -0.380 & 0.428 & 0.001 & 0.052 & 0.027 & 0.006 & 0.116 & -0.341 & 0.019 & -0.235 & 0.071 & -0.067 & -0.236 & 0.023 & -0.125 & $-0.640^{* *}$ \\
\hline & $\mathrm{p}_{\mathrm{g}}$ & 0.453 & -0.441 & -0.001 & 0.212 & -0.096 & -0.076 & -1.468 & 1.147 & 0.040 & 0.527 & 0.051 & -0.761 & -0.232 & -0.249 & 0.212 & $-0.681^{* *}$ \\
\hline \multirow[t]{2}{*}{$\overline{\mathrm{LC}}$} & $\mathrm{p}_{\mathrm{p}}$ & 0.500 & -0.523 & -0.031 & -0.056 & -0.028 & -0.010 & -0.081 & 0.203 & -0.031 & 0.294 & -0.121 & 0.103 & 0.378 & -0.028 & 0.200 & $0.769^{* *}$ \\
\hline & $\mathrm{p}_{\mathrm{g}}$ & -0.646 & 0.604 & 0.043 & -0.222 & 0.096 & 0.082 & 1.066 & -0.841 & -0.055 & -0.687 & -0.087 & 1.172 & 0.394 & 0.318 & -0.354 & $0.884^{* *}$ \\
\hline \multirow[t]{2}{*}{ GC } & $\mathrm{p}_{\mathrm{p}}$ & 0.511 & -0.553 & -0.028 & -0.053 & -0.028 & -0.012 & -0.090 & 0.217 & -0.025 & 0.370 & -0.142 & 0.102 & 0.367 & -0.040 & 0.240 & $0.835^{* *}$ \\
\hline & $\mathrm{p}_{\mathrm{g}}$ & -0.612 & 0.564 & 0.033 & -0.199 & 0.088 & 0.102 & 1.175 & -0.779 & -0.049 & -0.776 & -0.096 & 1.079 & 0.344 & 0.417 & -0.381 & $0.910^{* *}$ \\
\hline \multirow[t]{2}{*}{$\mathrm{KR} / \mathrm{C}$} & $\mathrm{p}_{\mathrm{p}}$ & 0.133 & -0.135 & -0.036 & -0.042 & -0.019 & -0.018 & -0.043 & 0.112 & -0.018 & 0.244 & -0.217 & 0.064 & 0.284 & -0.019 & 0.149 & $0.441^{* *}$ \\
\hline & $\mathrm{p}_{\mathrm{g}}$ & -0.164 & 0.128 & 0.049 & -0.161 & 0.061 & 0.159 & 0.552 & -0.429 & -0.035 & -0.545 & -0.136 & 0.719 & 0.274 & 0.207 & -0.232 & $0.447^{* *}$ \\
\hline \multirow[t]{2}{*}{$\bar{K} / \mathbf{R}$} & $\mathrm{p}_{\mathrm{p}}$ & 0.588 & -0.625 & -0.017 & -0.060 & -0.030 & -0.013 & -0.077 & 0.185 & -0.026 & 0.306 & -0.112 & 0.123 & 0.415 & -0.028 & 0.271 & $0.899^{* *}$ \\
\hline & $\mathrm{p}_{\mathrm{g}}$ & -0.664 & 0.605 & 0.020 & -0.234 & 0.097 & 0.118 & 1.046 & -0.703 & -0.052 & -0.675 & -0.079 & 1.242 & 0.373 & 0.282 & -0.410 & $0.965^{* *}$ \\
\hline \multirow[t]{2}{*}{$\mathrm{KN} / \mathrm{C}$} & $\mathrm{p}_{\mathrm{p}}$ & 0.543 & -0.569 & -0.021 & -0.061 & -0.030 & -0.015 & -0.078 & 0.189 & -0.028 & 0.318 & -0.144 & 0.120 & 0.428 & -0.027 & 0.257 & $0.882^{* *}$ \\
\hline & $\mathrm{p}_{\mathrm{g}}$ & -0.624 & 0.560 & 0.024 & -0.239 & 0.098 & 0.137 & 1.049 & -0.701 & -0.057 & -0.704 & 0.099 & 1.222 & 0.379 & 0.285 & -0.386 & $0.944^{* *}$ \\
\hline \multirow[t]{2}{*}{$\overline{\mathrm{KW}}$} & $\mathrm{p}_{\mathrm{p}}$ & 0.578 & -0.607 & -0.009 & -0.037 & -0.018 & -0.001 & -0.063 & 0.138 & -0.016 & 0.266 & -0.074 & 0.060 & 0.208 & -0.056 & 0.189 & $0.557^{* *}$ \\
\hline & $\mathrm{p}_{\mathrm{g}}$ & -0.728 & 0.651 & 0.013 & -0.158 & 0.059 & -0.006 & 0.926 & -0.531 & -0.033 & -0.603 & -0.053 & 0.651 & 0.201 & $\mathbf{0 . 5 3 7}$ & -0.323 & $0.605^{* *}$ \\
\hline \multirow[t]{2}{*}{ S \% } & $\mathrm{p}_{\mathrm{p}}$ & 0.661 & -0.739 & -0.023 & -0.051 & -0.028 & -0.013 & -0.056 & -0.124 & -0.018 & 0.259 & -0.094 & 0.097 & 0.321 & -0.031 & 0.343 & $0.752^{* *}$ \\
\hline & $\mathrm{p}_{\mathrm{g}}$ & -0.843 & 0.821 & 0.030 & -0.231 & 0.106 & 0.149 & 0.838 & -0.503 & -0.040 & -0.609 & -0.065 & 1.051 & 0.302 & 0.357 & -0.485 & $0.877^{* *}$ \\
\hline
\end{tabular}

Residual effect: $\mathrm{p}_{\mathrm{p}(\mathrm{R})}=0.285 \& \mathrm{R}_{\mathrm{p}}{ }^{2}(\%)=91.87$

Residual effect: $\mathrm{p}_{\mathrm{g}(\mathrm{R})}=0.502 \& \mathrm{R}_{\mathrm{g}}{ }^{2}(\%)=74.83$ 


\section{Character association}

Correlation studies revealed that genotypic correlation coefficients were higher than phenotypic correlation coefficients for most of the characters under study indicating the strong inherent association between the characters which governed largely by genetic causes (strong coupling linkage) and generally less subjected to environmental forces. This was in agreement with many earlier findings of Ajaz A. Lone et al., 2010, Pavan et al., 2011, Begum et al., 2016, Sayo Sesay et al., 2017 and Usha Rani et al., 2017. The correlation coefficients both at phenotypic $\left(r_{p}\right)$ and genotypic $\left(r_{g}\right)$ levels were presented below in table 3 .

Yield was positively and significantly correlated with plant height, ear height, length of cob, girth of cob, number of kernel rows per cob, number of kernels per row, number of kernels per cob, 100 kernel weight and shelling\% at the phenotypic level. At the genotypic level almost similar trend was observed. Ajaz A. Lone et al., 2010 showed that yield was positively correlated with plant height $\left(r_{\mathrm{g}}=0.267\right)$, cob length and diameter and 100 kernel weight $\left(r_{\mathrm{g}}=0.322\right)$ under ESM conditions. Nataraj et al., 2014 reported that grain yield had positive and significant correlation with ear length and diameter, number of kernels per row, number of kernel rows per ear, ear and plant height, 100 grain weight both at genotypic and phenotypic levels. Usha Rani et al., 2017 found yield to be significantly and positively associated with cob length $\left(0.7866^{* *} ; 0.3864^{* *}\right)$, number of kernel rows per cob $\left(0.4066^{* * ;} ; 0.4272^{* *}\right)$ and 100 grain weight $(0.7692 * * ; 0.4160 * *)$ both at phenotypic and genotypic level. It was revealed that all the significantly and positively correlated yield components were highly inter-correlated among themselves. So, intensive selection in the positive side of any one of these traits would automatically improve other traits, thus, increasing the yield.

\section{Path co-efficient analysis}

The traits having high positive correlation along with high direct effects were expected to be useful as selection criteria in improvement program. In the present study, number of kernels per row and number of kernels per cob had higher indirect positive correlation with yield and also direct positive effect with yield. The findings were in accordance with Pavan et al., 2011 for number of kernel rows per cob and number of kernels per row and also with that of Begum et al., 2016 for number of kernel rows per cob that had highly significant positive direct effect on yield.

Plant height had positive correlation with yield but direct negative effect on grain yield and higher indirect positive effect via days to $50 \%$ tasseling at phenotypic level and senescence per cent at genotypic level. Similar results were reported by Sayo Sesay et al., 2017. Similarly, length of cob had positive correlation with grain yield but direct negative effect and higher indirect positive effect via days to $50 \%$ tasseling at phenotypic level and number of kernels per row at genotypic level. Characters like days to $50 \%$ tasseling, days to $50 \%$ silking, senescence percent and difference in senescence per cent showed negative correlation with yield. Hence, hybrids with smaller magnitude of these characters should be selected. Table 4 presented the direct and indirect effects of fifteen traits on grain yield at phenotypic level.

\section{References}

Ajaz A. Lone, Warsi M.Z.K., Nehvi F A and Dar S A. 2010. Studies on Character Association in Winter Maize under 
Normal and Excess Soil Moisture (ESM) Conditions. Maize Genetics Cooperation Newsletter. 84.

Begum S, Ahmed A, Omy S. H, Rohman M. M and Amiruzzaman M. 2016. Genetic variability, character Association and Path Analysis in Maize (Zea mays L.). Bangladesh Journal of Agrilcultural Research. 41(1): 173-182.

DMR, 2001. In: Azad, C.S. (Ed.), Proceedings of the 49th Annual Maize Workshop, Directorate of Maize Research (DMR). University of Agriculture and Technology, Kanpur (UP), India, 5-9 April 2001.

Nataraj V, Shahi J. P, Agarwal V. 2014. Correlation and Path Analysis in Certain Inbred Genotypes of Maize (Zea Mays L.) at Varanasi. International Journal of Innovative Research and Development, 3 (1):14-17.

Nzuve F, Githiri S, Mukunya D.M. and Gethi J. 2014. Genetic Variability and Correlation Studies of Grain Yield and Related Agronomic Traits in Maize. Journal of Agricultural Science, 6(9): 18-23.

Sayo Sesay, David Kolawole Ojo, Omolayo Johnson Ariyo, Silvestro Meseka, Lawrence Stephen Fayeun, Ayoola
Oluwaseun Omikunle and Adebiyi Oyeboade Oyetunde. 2017. Correlation and path coefficient analysis of topcross and three-way cross hybrid maize populations. African Journal of Agricultural Research. 12(10): 780-789.

Sravanti K, Swarnalatha Devi I, Sudarshan M.R and Supriya K. 2017. Evaluation of Maize genotypes (Zea mays L.) for variability, heritability and genetic advance. International Journal of Current Microbioliology and Applied Sciences. 6(10): 2227-2232.

Usha Rani G, Satyanarayana Rao V, Lal Ahmad M. and Narasimha Rao K.L. 2017.Character Association and Path Coefficient Analysis of Grain Yield and Yield Components in Maize (Zea mays L.). International Journal of Current Microbiology and Applied Sciences, 6(12): 4044-4050.

Yogesh Pandey, Vyas R. P, Jaydev Kumar, Lokendra Singh, Singh H. C, Yadav P. $\mathrm{C}$ and Vishwanath. 2017. Heritability, Correlation and Path Coefficient Analysis for Determining Interrelationships among Grain Yield and Related Characters in Maize (Zea mays L). International Journal of Pure and Applied Bioscience. 5(2): 595-603.

\section{How to cite this article:}

Modekurti Sravana Lakshmi, Prem Narayan Jagadev, Sindhusnata Das, Devraj Lenka, Digvijay Swain and Swapan K. Tripathy. 2018. Genetic Variability and Association Analysis of Maize Hybrids under Excessive Soil Moisture Condition. Int.J.Curr.Microbiol.App.Sci. 7(09): 29352941. doi: https://doi.org/10.20546/ijcmas.2018.709.365 